\title{
Bioactive $\alpha$-pyrone meroterpenoids from mangrove endophytic fungus Penicillium sp.
}

\author{
Bo Ding ${ }^{1,2}$, Zhiyuan Wang ${ }^{2}$, Xishan Huang ${ }^{1}$, Yayue Liu ${ }^{1}$ Wenrui \\ Chen ${ }^{2, *}$ and Zhigang She ${ }^{1, *}$ \\ ${ }^{1}$ School of Chemistry and Chemical Engineering, Sun Yat-sen University, Guangzhou, \\ China \\ ${ }^{2}$ Guangdong Inspection and Quarantine Technology Center, Guangzhou, China
}

Five $\alpha$-pyrone meroterpenoids, including one new 3-epiarigsugacin E (1) and four known compounds, arisugacin D (2), arisugacin B (3), territrem C (4) and terreulactone C (5) were obtained from marine fungus Penicillium sp. SK5GW1L. Their structures were identified by MS and NMR experiments, and the absolute configuration of compound $\mathbf{1}$ was further confirmed by low temperature $(150 \mathrm{~K})$ single crystal X-ray diffraction with $\mathrm{Cu} \mathrm{K} \alpha$ radiation. Compounds $\mathbf{3}, \mathbf{4}$ and $\mathbf{5}$ showed strong inhibitory activities against acetylcholinesterase (AchE) with $\mathrm{IC}_{50}$ values of $3.03 \mu \mathrm{M}, 0.23 \mu \mathrm{M}$ and $0.028 \mu \mathrm{M}$, respectively. 


\section{Support Information}

Table S1 ${ }^{1} \mathrm{H}$ NMR (500 MHz), ${ }^{13} \mathrm{C}$ NMR (125M), and 2D- NMR data for compound 1 (ppm from TMS, $J=\mathrm{Hz}$, in $\mathrm{CDCl}_{3}$ ).

Table S2. The single crystal parameters of compound 1

Figure S1. The single crystal structure of compound $\mathbf{1}$

Figure S2. ${ }^{1} \mathrm{H}$ and ${ }^{13} \mathrm{C}$ NMR, HSQC, and HMBC data for compound 1

Figure S3. UV of compound 1

Figure S4. IR of compound 1

Figure S5. ESIMS of compound 1

Figure S6. HRESIMS of compound 1

The single crystal cif file of compound $\mathbf{1}$ 
Table S1 ${ }^{1} \mathrm{H}$ NMR (500 MHz), ${ }^{13} \mathrm{C}$ NMR (125M), and 2D- NMR data for compound $\mathbf{1}$ (ppm from TMS, $J=\mathrm{Hz}$, in $\mathrm{CDCl}_{3}$ ).

\begin{tabular}{|c|c|c|c|c|}
\hline No. & $\delta_{\mathrm{C}}$ & $\delta_{\mathrm{H}}$ & ${ }^{1} \mathrm{H}-{ }^{1} \mathrm{H} \mathrm{COSY}$ & $\operatorname{HMBC}(\mathrm{H} \rightarrow \mathrm{C})$ \\
\hline \multirow[t]{2}{*}{1} & 30.9 & $1.67-1.65, \mathrm{~m}$ & & $2,10,19,3,5$ \\
\hline & & $1.45, \mathrm{dd}(8.5,2.3)$ & & \\
\hline \multirow[t]{2}{*}{2} & 27.4 & $1.79-1.76, \mathrm{~m}$ & $\mathrm{H}-3$ & $1,10,4,3$ \\
\hline & & $1.68-1.65, \mathrm{~m}$ & & \\
\hline 3 & 73.8 & $3.88, \mathrm{dd}(11.0,5.7)$ & $\mathrm{H}-2$ & 17,18 \\
\hline 4 & 43.9 & & & \\
\hline 5 & 78.1 & & & \\
\hline \multirow[t]{2}{*}{6} & 25.3 & $1.91-1.87, \mathrm{~m}$ & $\mathrm{H}-7$ & $7,5,8$ \\
\hline & & $1.71-1.69, \mathrm{~m}$ & & \\
\hline \multirow[t]{2}{*}{7} & 33.9 & $2.18-2.14, \mathrm{~m}$ & $\mathrm{H}-6$ & $20,6,9,8$ \\
\hline & & $1.89-1.86, \mathrm{~m}$ & & \\
\hline 8 & 80.2 & & & \\
\hline 9 & 43.1 & $2.46, \mathrm{dd}(13.1,4.7)$ & $\mathrm{H}-11$ & $20,11,1,10,8$ \\
\hline 10 & 40.6 & & & \\
\hline \multirow[t]{2}{*}{11} & 17.4 & 2.39, dd $(16.7,4.7)$ & H-9 & $9,8,12,13$ \\
\hline & & $2.21-2.24, \mathrm{~m}$ & & \\
\hline 12 & 98.8 & & & \\
\hline 13 & 163.7 & & & \\
\hline 14 & 97.0 & $6.24, \mathrm{~s}$ & & $12,1^{\prime}, 15,13$ \\
\hline 15 & 158.4 & & & \\
\hline 16 & 164.8 & & & \\
\hline 1 ' & 124.2 & & & \\
\hline $2^{\prime}$ & 127.1 & 7.73, d (8.8) & H-3' & $15,4^{\prime}, 6^{\prime}$ \\
\hline 3 ' & 114.3 & $6.93, \mathrm{~d}(8.8)$ & H-2' & $1^{\prime}, 4^{\prime}, 2^{\prime}$ \\
\hline $4^{\prime}$ & 158.4 & & & \\
\hline 5 & 114.3 & $6.93, \mathrm{~d}(8.8)$ & H-6 & $1^{\prime}, 4^{\prime}, 6^{\prime}$ \\
\hline 6 & 127.1 & $7.73, \mathrm{~d}(8.8)$ & $\mathrm{H}-5$ & $15,2^{\prime}, 4^{\prime}$ \\
\hline 17 & 17.6 & $0.96, \mathrm{~s}$ & & $18,4,3,5$ \\
\hline 18 & 22.8 & $1.09, \mathrm{~s}$ & & $17,4,3,5$ \\
\hline 19 & 18.4 & $1.06, \mathrm{~s}$ & & $1,10,9,5$ \\
\hline $4^{\prime}-\mathrm{OCH}_{3}$ & 55.6 & $3.86, \mathrm{~s}$ & & $4^{\prime}$ \\
\hline 20 & 21.2 & $1.28, \mathrm{~s}$ & & $8,9,7,10,6$ \\
\hline
\end{tabular}


Table S2. The single crystal parameters of compound $\mathbf{1}$

crystallographic data of compound $\mathbf{1}$

\begin{tabular}{|c|c|c|c|}
\hline parameter & parameter values & parameter & parameter values \\
\hline Empirical formula & $\mathrm{C}_{27} \mathrm{H}_{34} \mathrm{O}_{6}$ & Reflections & $15932 / 4078[\mathrm{R}(\mathrm{int})=0.02$ \\
\hline Formula weight & 454.54 & \multirow[t]{2}{*}{ collected/unique } & \multirow[t]{2}{*}{ 48] } \\
\hline Temperature & $150(2) \mathrm{K}$ & & \\
\hline Wavelength & $1.54178 \stackrel{0}{A}$ & \multirow[t]{2}{*}{ Absorption correction } & Semi-empirical from \\
\hline Crystal system, space group & Orthorhombic, P 212121 & & Equivalents \\
\hline Unit cell dimensions & & Max./min.transmission & $0.8726 / 0.7470$ \\
\hline $\mathrm{a}$ & $10.70160(10) \stackrel{0}{A} \alpha=90^{0}$ & \multirow[t]{2}{*}{ Refinement method } & Full-matrix \\
\hline $\mathrm{b}$ & $10.75190(10) \stackrel{0}{A}, \beta=90^{0}$ & & least-squares on $\mathrm{F}^{2}$ \\
\hline $\mathrm{c}$ & 20.1484(2) $\stackrel{0}{A}, \gamma=90^{0}$ & Data/restraints/paramet & \multirow[t]{2}{*}{$4078 / 0 / 305$} \\
\hline Volume & $2318.33(4) \stackrel{0}{A}^{3}$ & ers & \\
\hline Z, calculated density & $4,1.302 \mathrm{mg} / \mathrm{m}^{3}$ & Goodness-of-fit on $\mathrm{F}^{2}$ & 1.093 \\
\hline Absorption coefficient & $0.737 \mathrm{~mm}^{-1}$ & Final $\quad \mathrm{R} \quad$ indices & \multirow[t]{2}{*}{$\mathrm{R} 1=0.0297 \mathrm{WR} 2=0.0742$} \\
\hline $\mathrm{F}(000)$ & 976 & {$[\mathrm{I}>2 \operatorname{sigma}(\mathrm{I})]$} & \\
\hline Crystal size & $0.40 \times 0.32 \times 0.19 \mathrm{~mm}$ & $\mathrm{R}$ indices (all data) & $\mathrm{R} 1=0.0320 \mathrm{R} 2=0.0765$ \\
\hline Theta range for data collection & $4.39-66.91^{0}$ & \multirow{4}{*}{$\begin{array}{l}\text { Absolute structure } \\
\text { parameter } \\
\text { Largest diff. peak and } \\
\text { hole }\end{array}$} & $-0.13(13)$ \\
\hline Limiting indices & $-12 \leq \mathrm{h} \leq 12,-12 \leq \mathrm{k} \leq 12$ & & \multirow{3}{*}{0.133 and -0.196 e. $\stackrel{0}{A}^{-3}$} \\
\hline & $-23 \leq 1 \leq 22$ & & \\
\hline completeness to theta $=62.77$ & $99.3 \%$ & & \\
\hline
\end{tabular}

Figure S1. The single crystal structure of compound 1

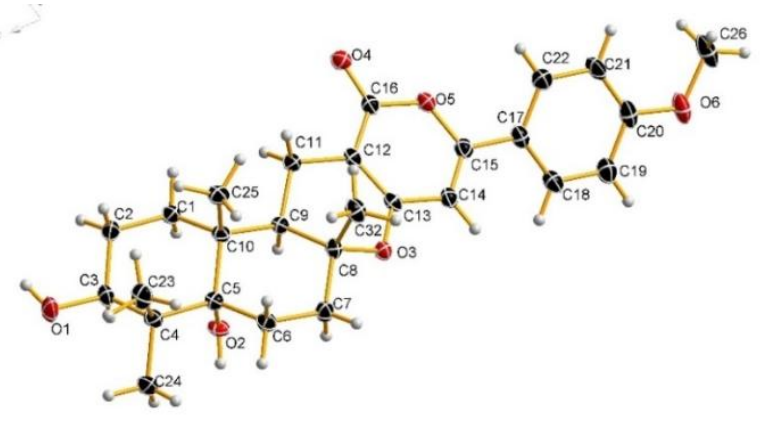


Figure S2. ${ }^{1} \mathrm{H}$ and ${ }^{13} \mathrm{C}$ NMR, COSY, HSQC, HMBC and NOE data for compound 1

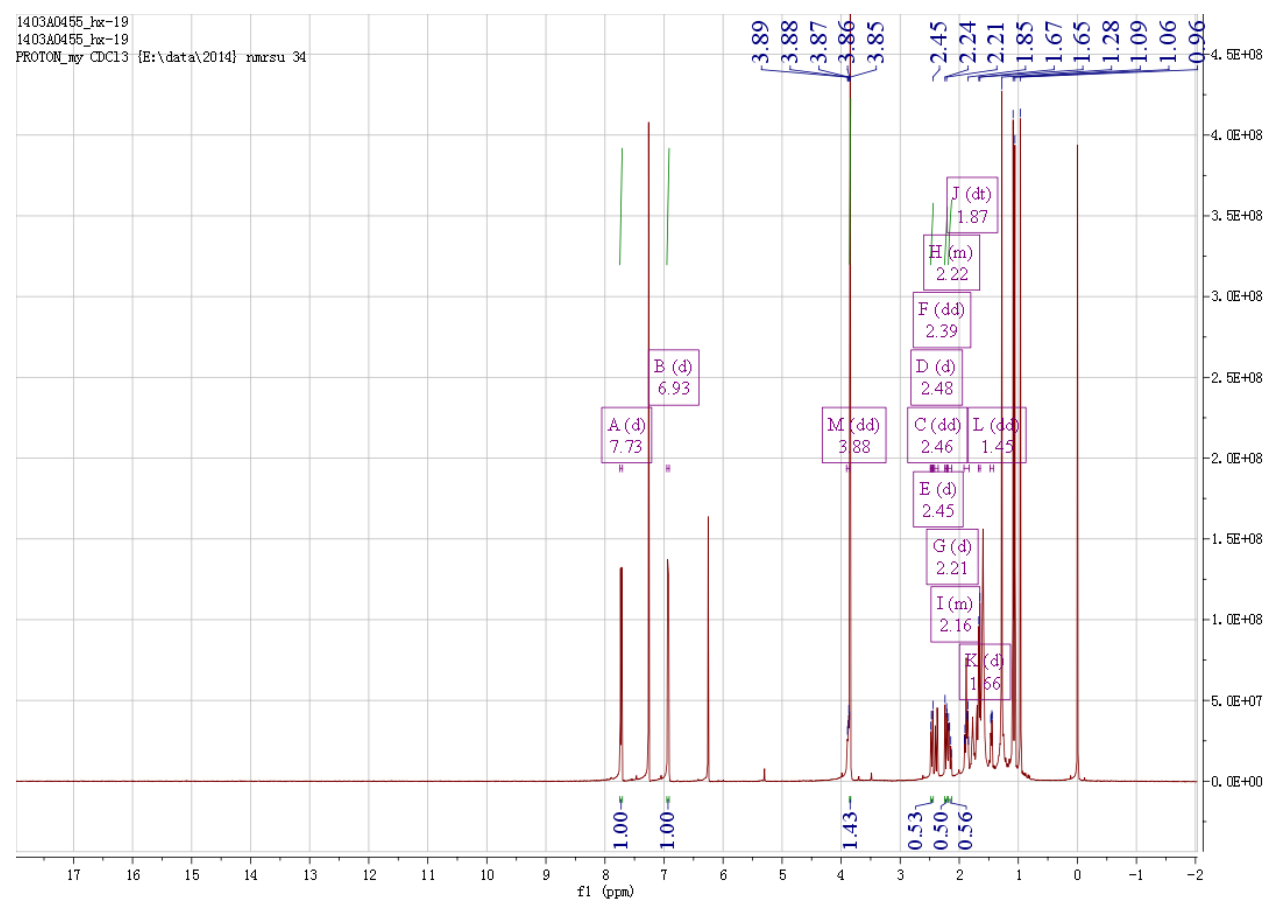

${ }^{1} \mathrm{H}-\mathrm{NMR}$ 


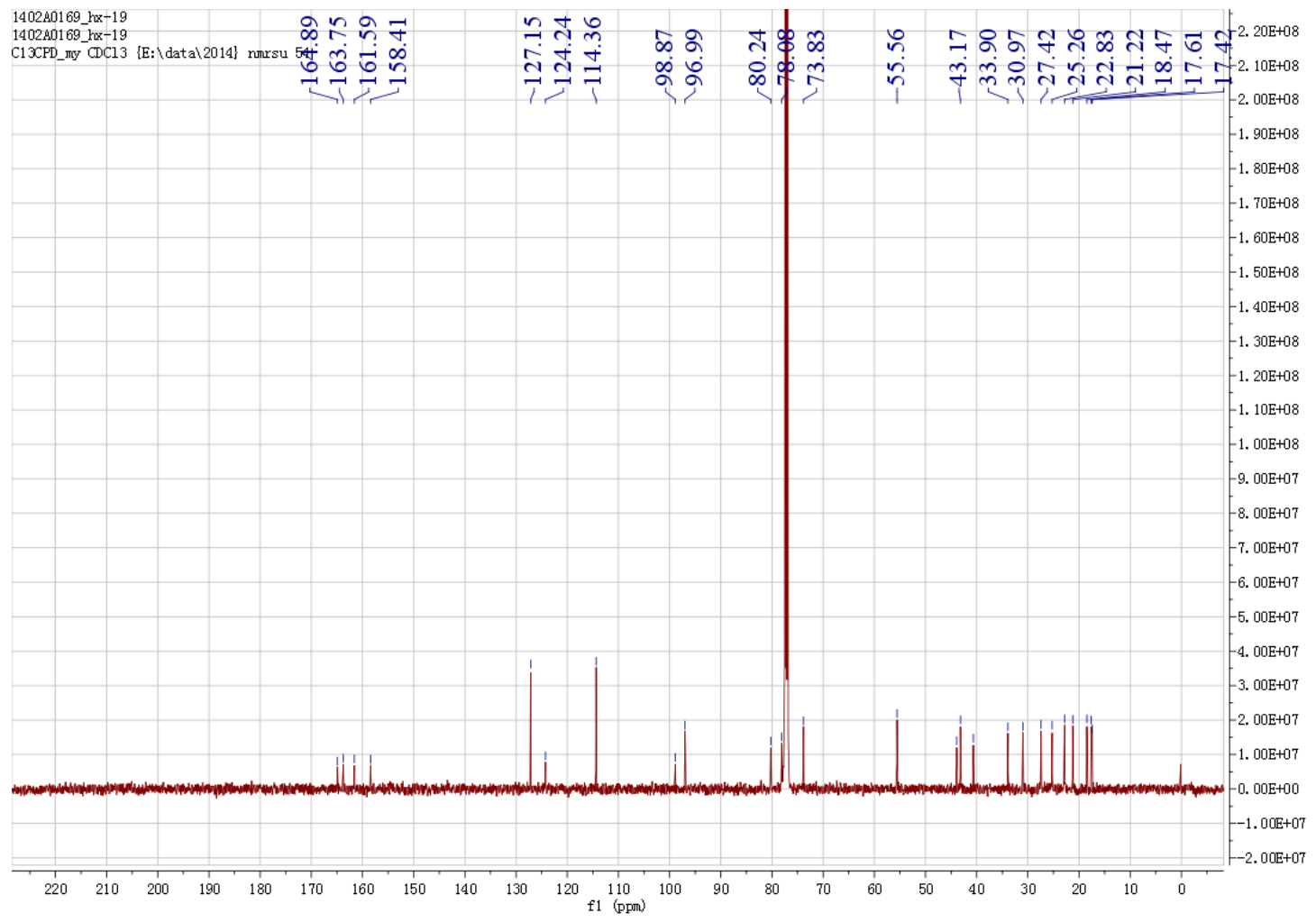

\section{${ }^{13}$ C NMR}

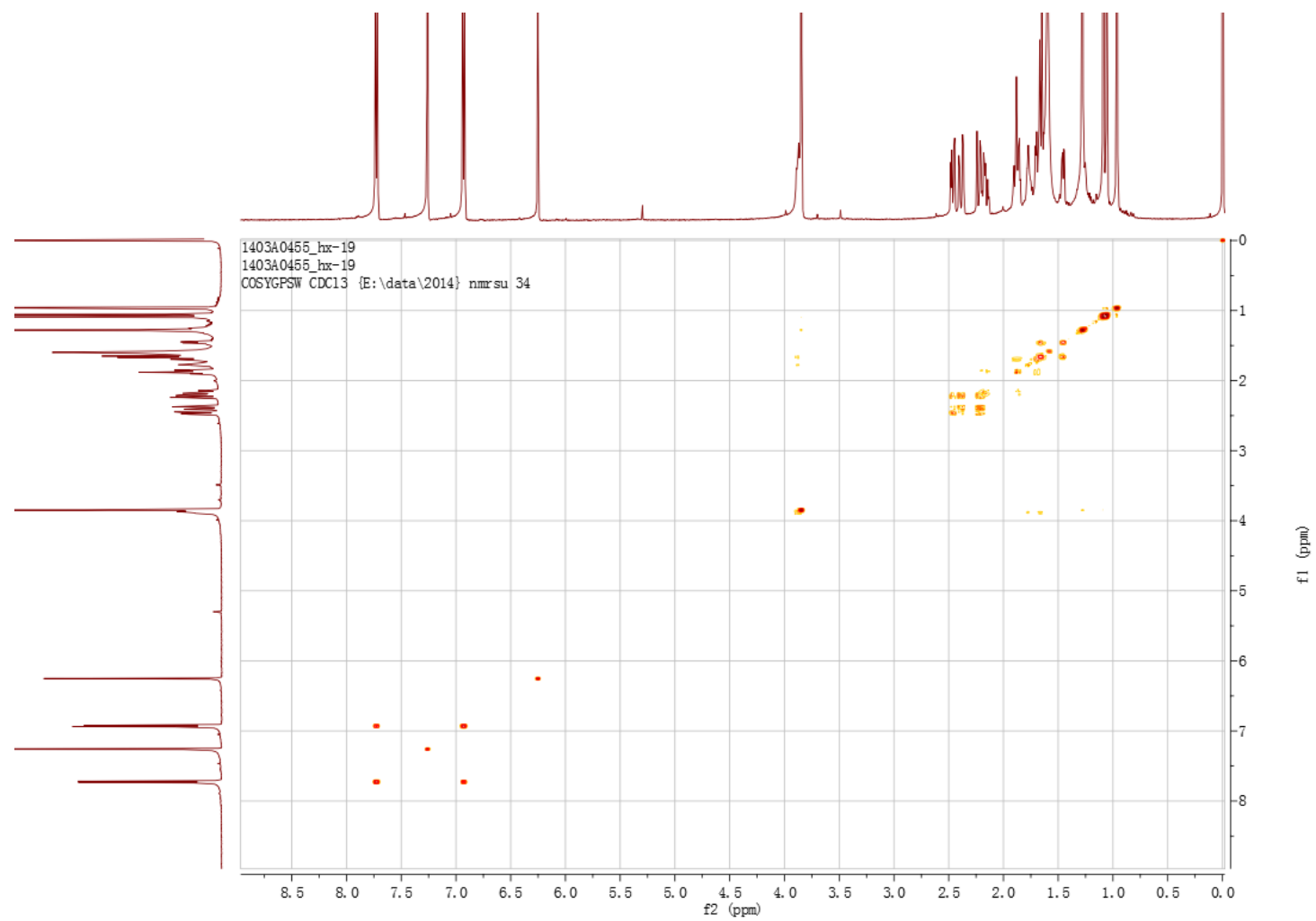

\section{${ }^{1} \mathrm{H}-{ }^{1} \mathrm{H} \mathrm{COSY}$}




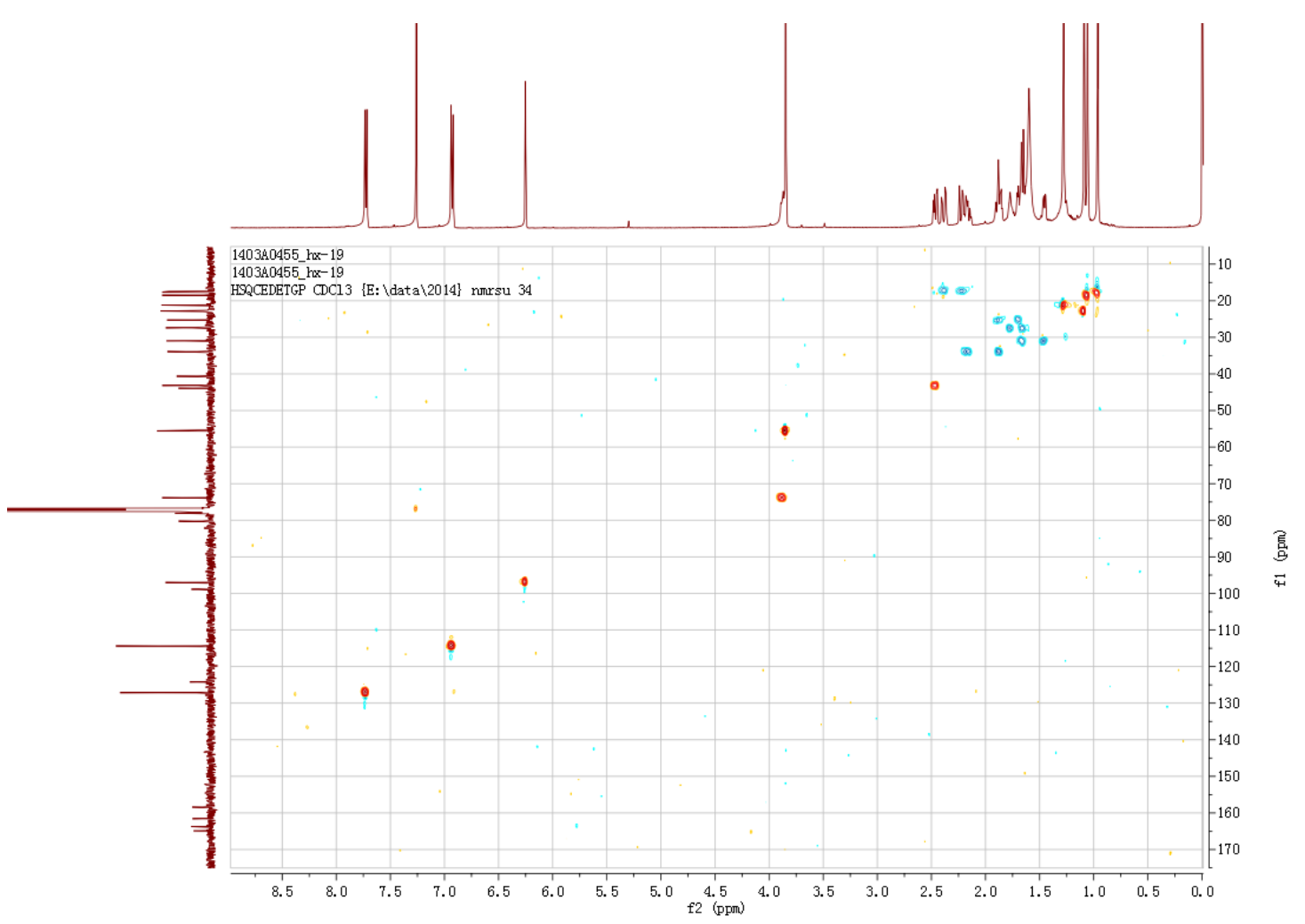

HSQC

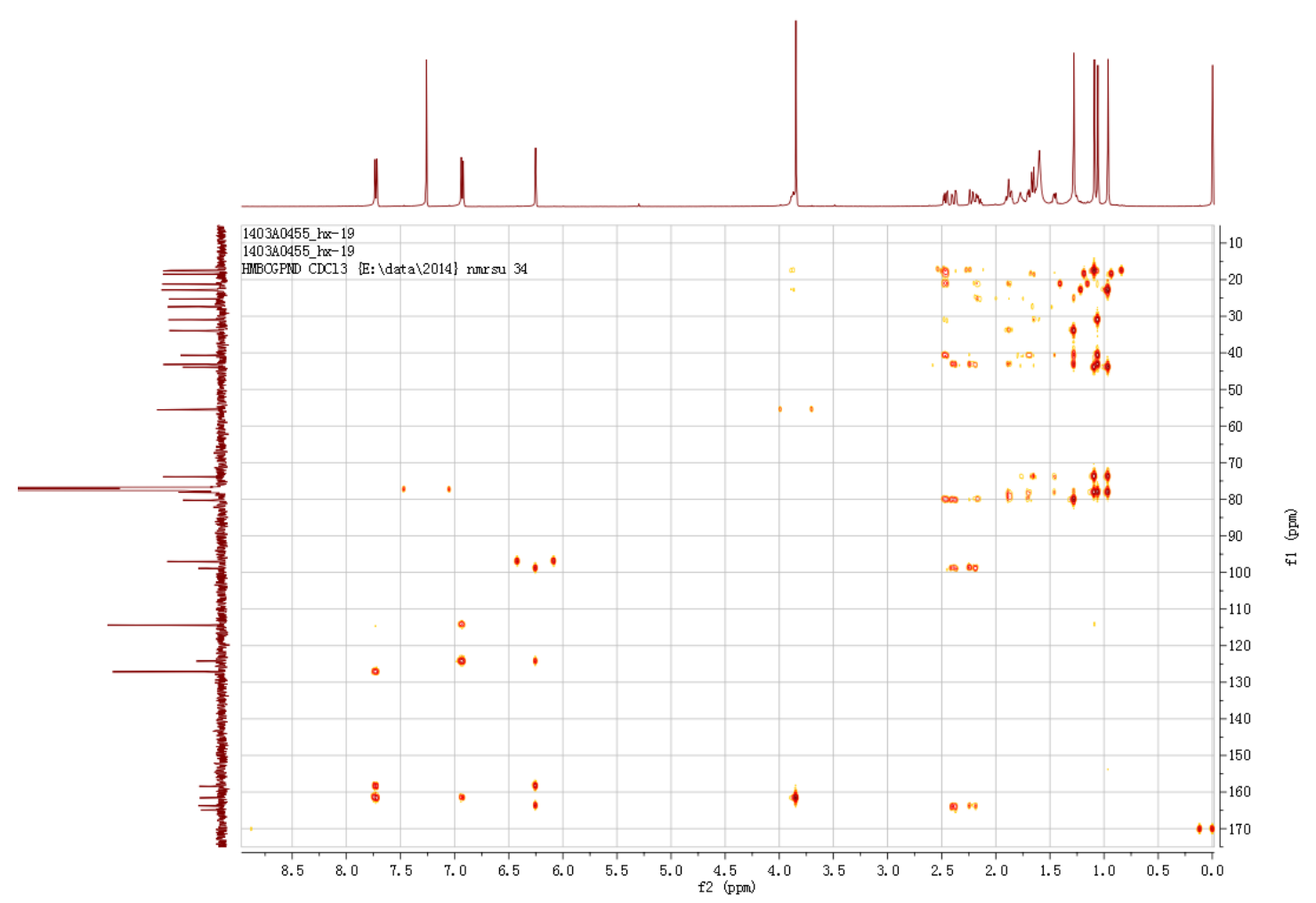

HMBC 


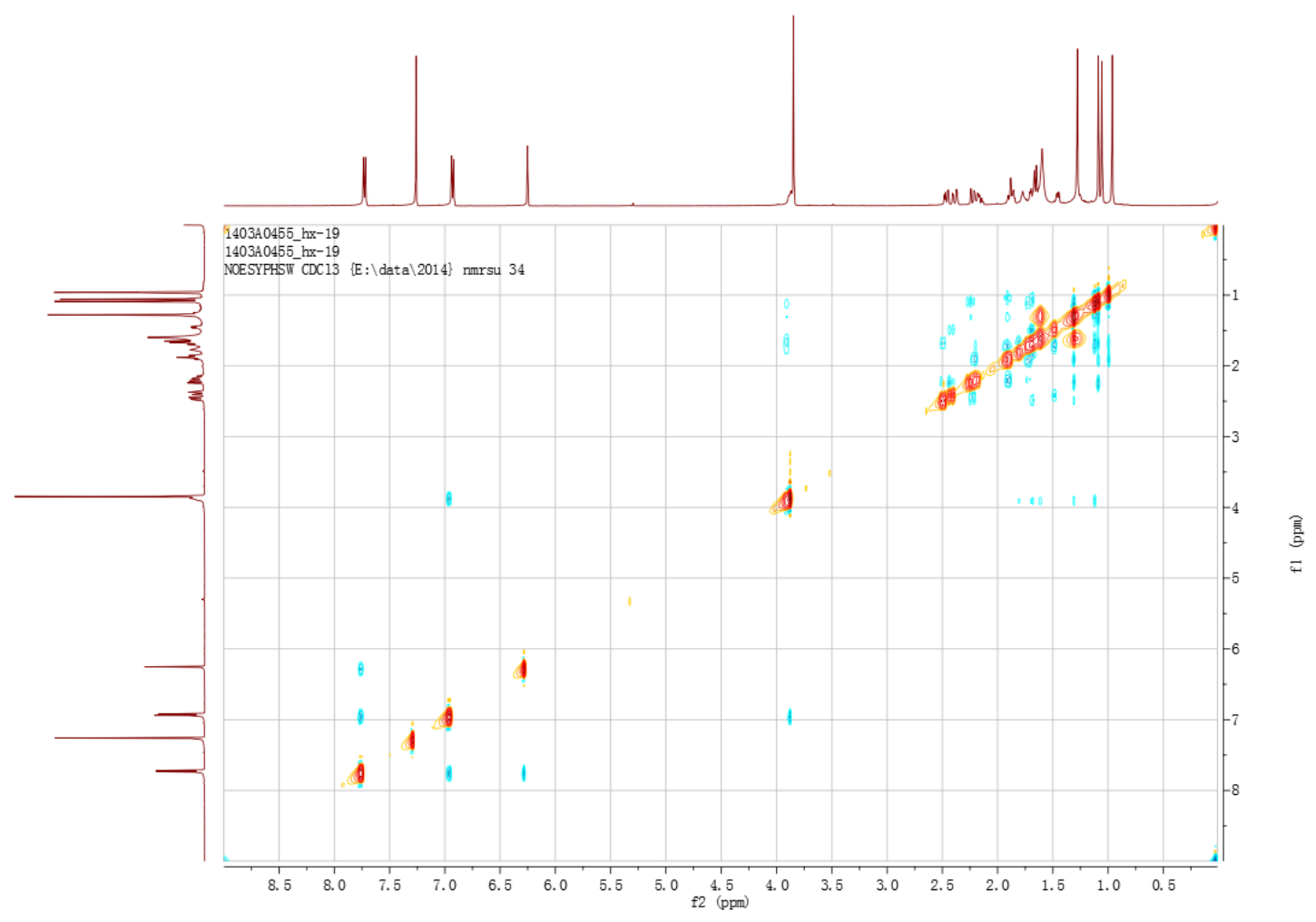

NOE

Figure S3. UV of compound 1

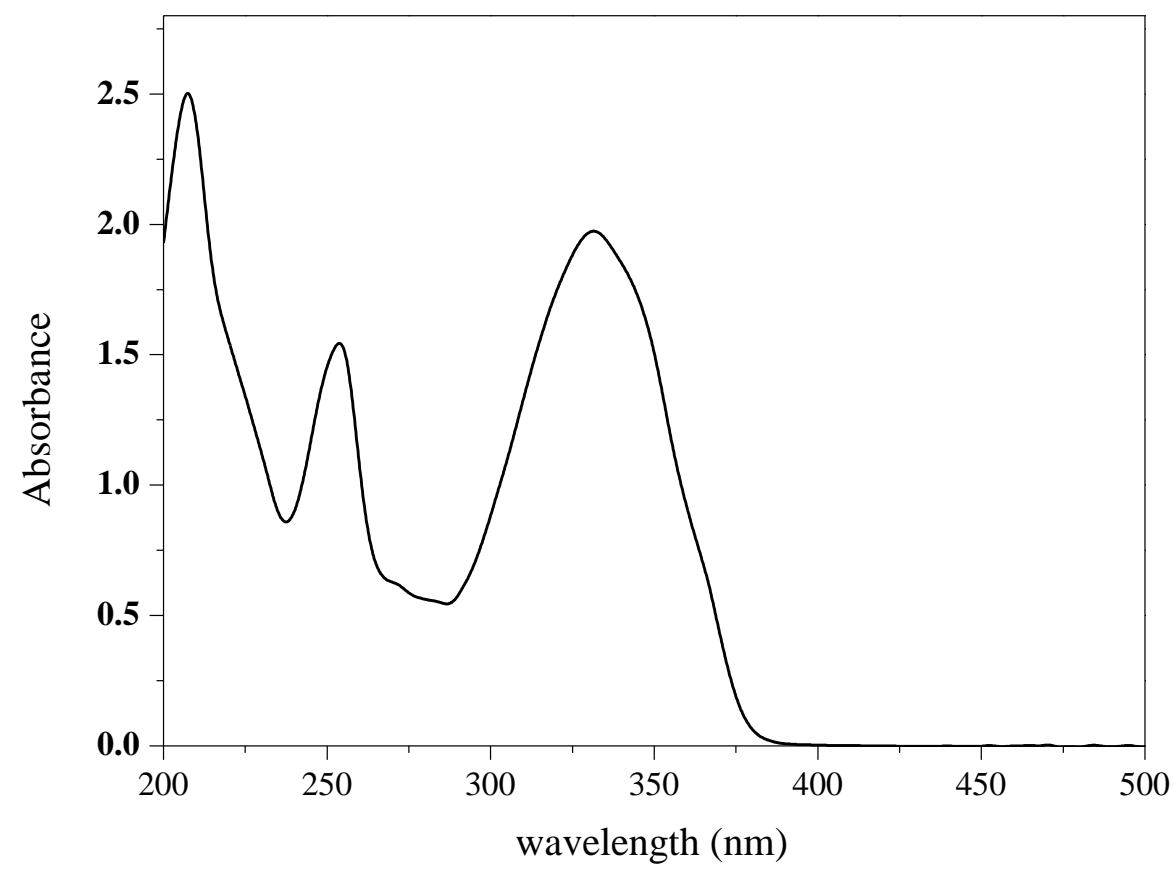


Figure S4. IR of compound 1

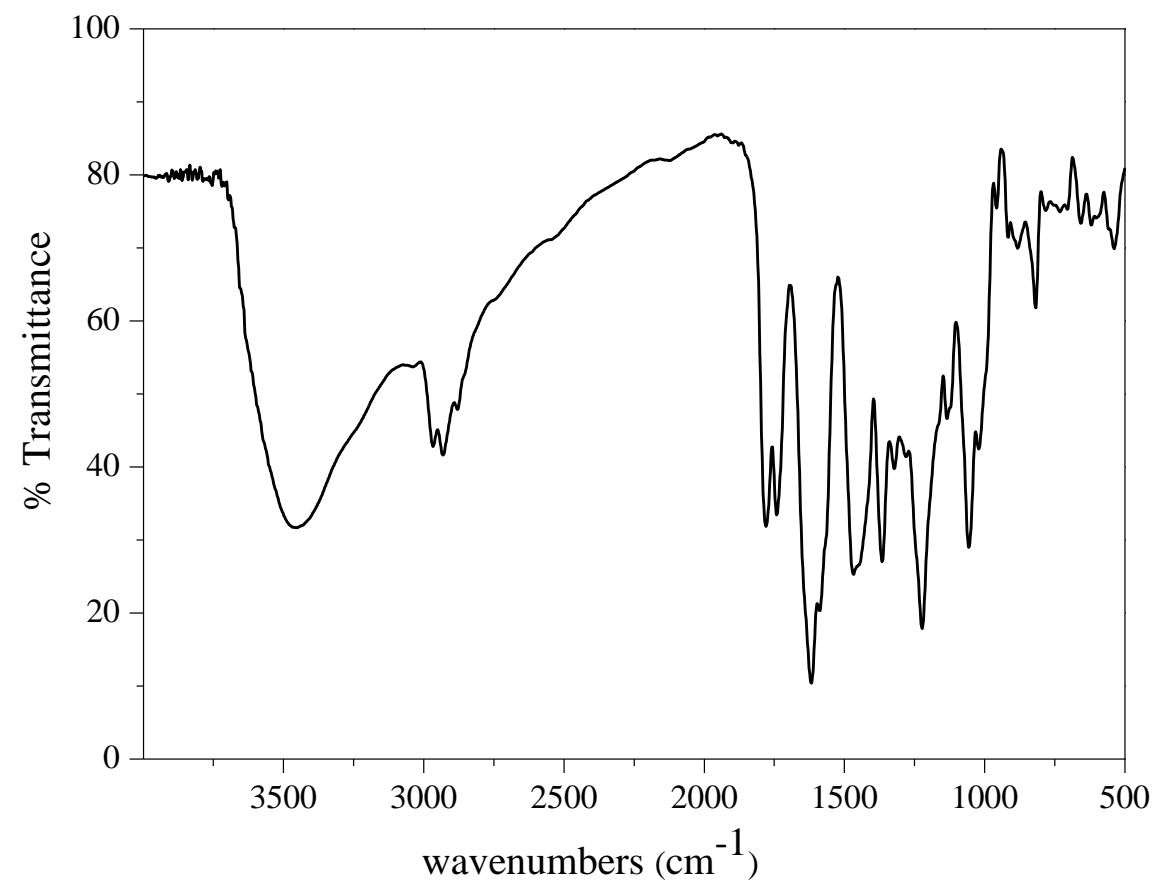

Figure S5. ESIMS of compound 1

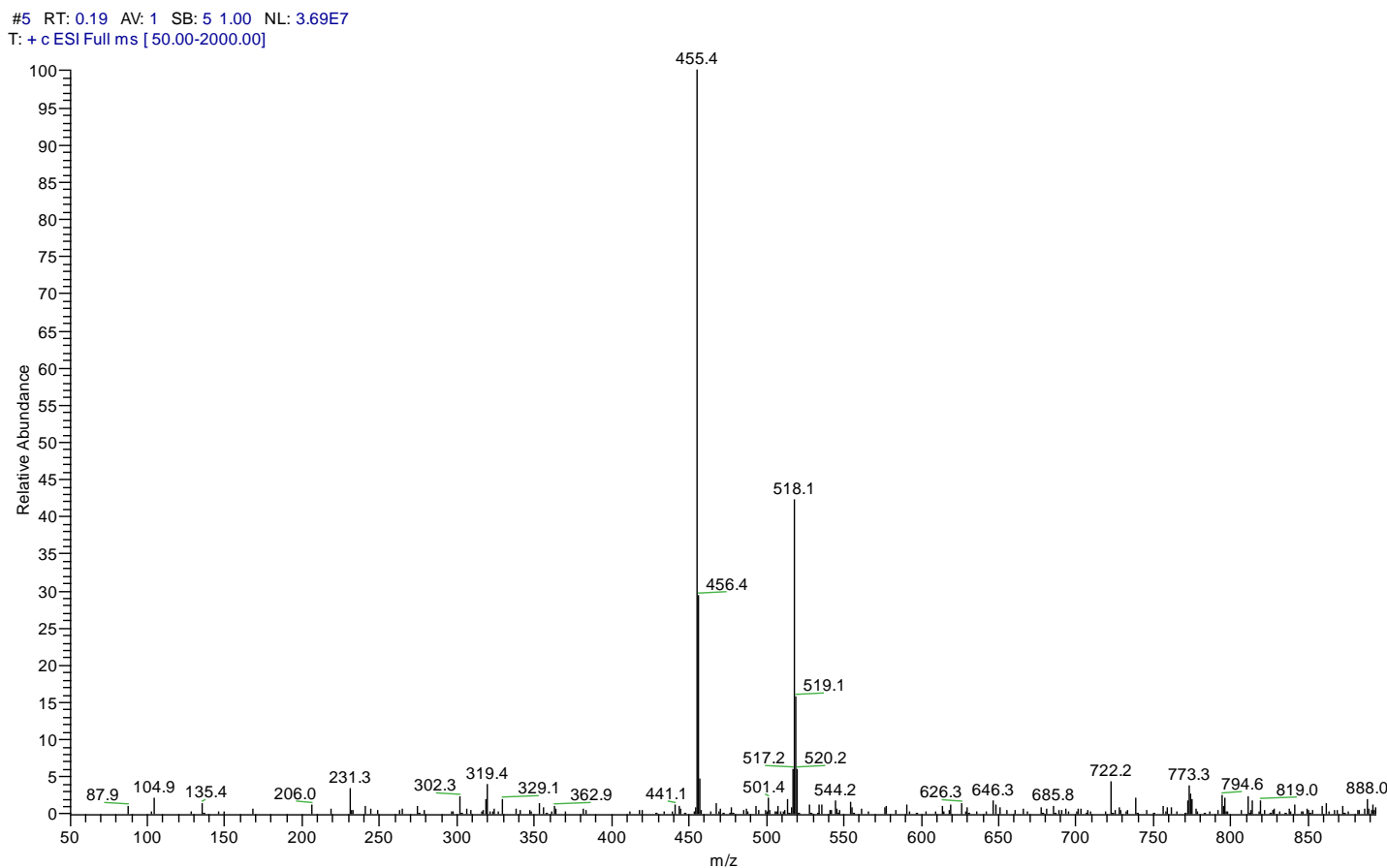


Figure S6. HRESIMS of compound 1

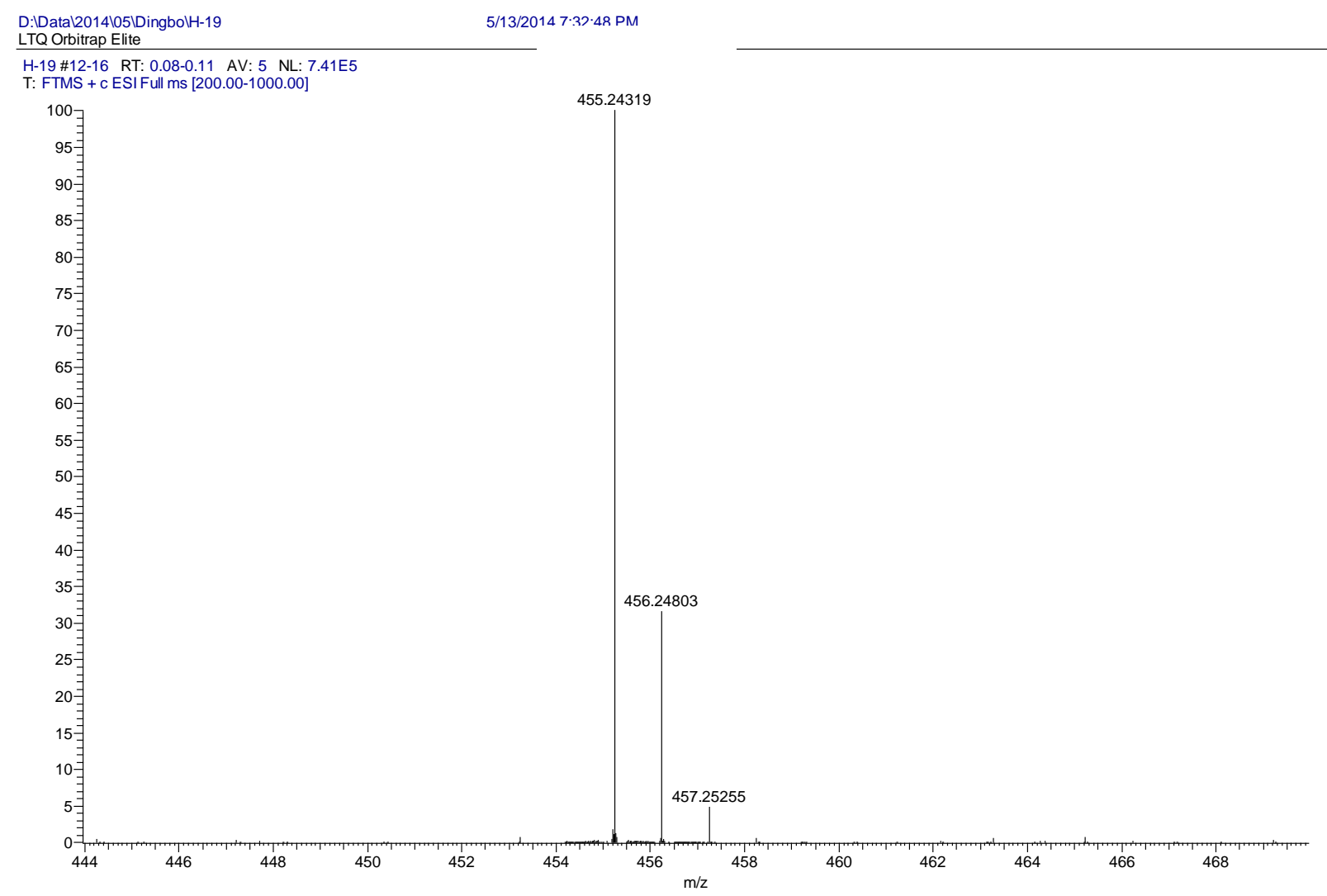

SPECTRUM - simulation :

$\mathrm{m} / \mathrm{z}$ Theo. Mass Delta $(\mathrm{ppm})$ RDB equiv. Composition

\section{Limits:}

(1) Charge: 1

(2) Nitrogen-Rule: Do not use

(3) Mass tolerance: $10.00 \mathrm{ppm}$

(4) Elements in use: ${ }^{12} \mathrm{C}\left(0^{\sim 30}\right),{ }^{1} \mathrm{H}\left(0^{\sim 60}\right),{ }^{16} \mathrm{O}\left(0^{\sim 15}\right)$; 
The single crystal cif file of compound $\mathbf{1}$

data_a

_audit_creation_method

SHELXL-97

_chemical_name_systematic

;

$?$

;

_chemical_name_common

?

_chemical_melting_point

?

_chemical_formula_moiety

?

_chemical_formula_sum

'C27 H34 O6'

_chemical_formula_weight

454.54

_chemical_absolute_configuration ad

loop_

_atom_type_symbol

_atom_type_description

_atom_type_scat_dispersion_real

_atom_type_scat_dispersion_imag

_atom_type_scat_source

$\begin{array}{llll}\mathrm{C}^{\prime} & \text { 'C' } & 0.0181 & 0.0091\end{array}$

'International Tables Vol C Tables 4.2.6.8 and 6.1.1.4'

$\begin{array}{llll} & \text { 'H' 'H' } \quad 0.0000 \quad 0.0000\end{array}$

'International Tables Vol C Tables 4.2.6.8 and 6.1.1.4'

$\begin{array}{llll}\text { 'O' } & \text { 'O' } & 0.0492 & 0.0322\end{array}$

'International Tables Vol C Tables 4.2.6.8 and 6.1.1.4'

_symmetry_cell_setting 'Orthorhombic'

_symmetry_space_group_name_H-M 'P2(1)2(1)2(1) '

loop_

_symmetry_equiv_pos_as_xyz

'x, y, z'

$'-x+1 / 2,-y, z+1 / 2$ '

$'-x, y+1 / 2,-z+1 / 2$ '

'x+1/2, -y+1/2, -z'

_cell_length_a

10.70160(10)

_cell_length_b

10.75190(10)

_cell_length_c

20.1484(2)

_cell_angle_alpha

90.00 


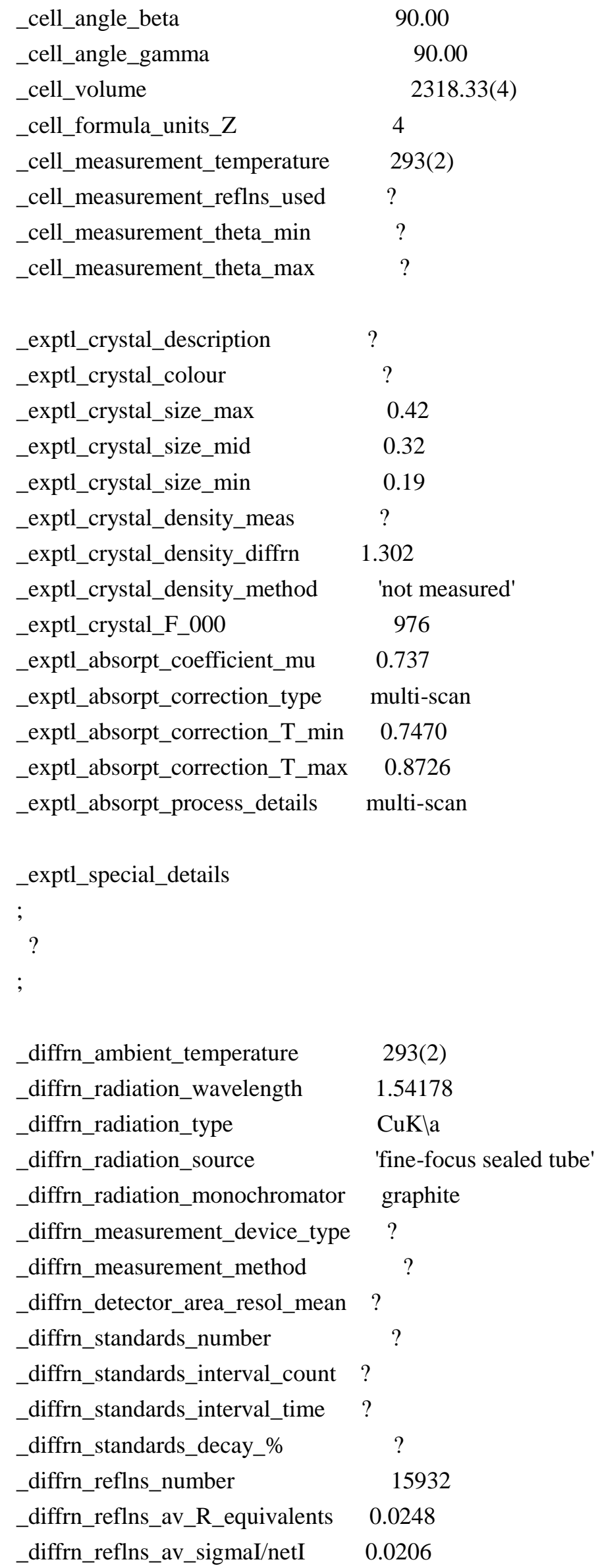




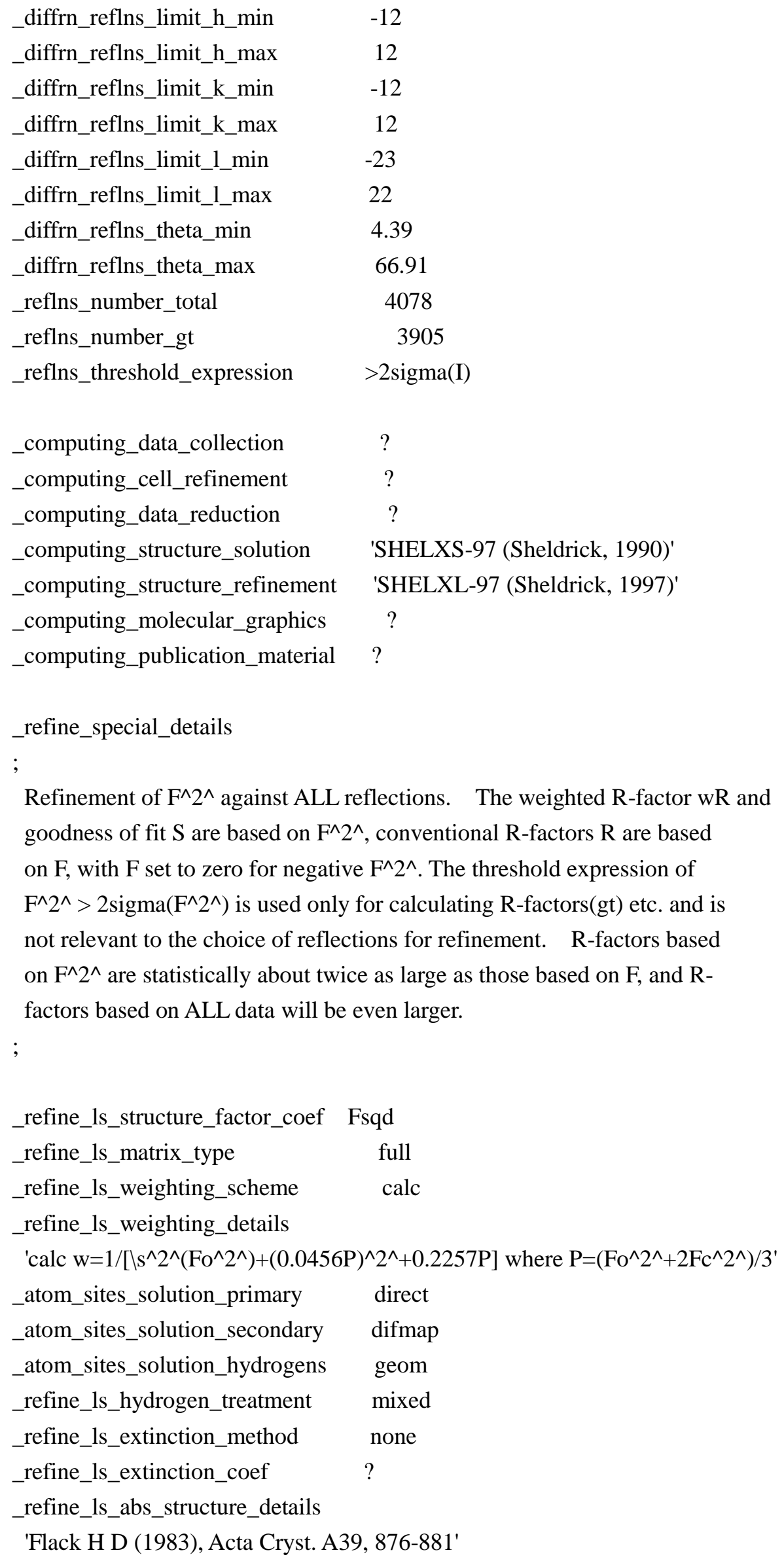




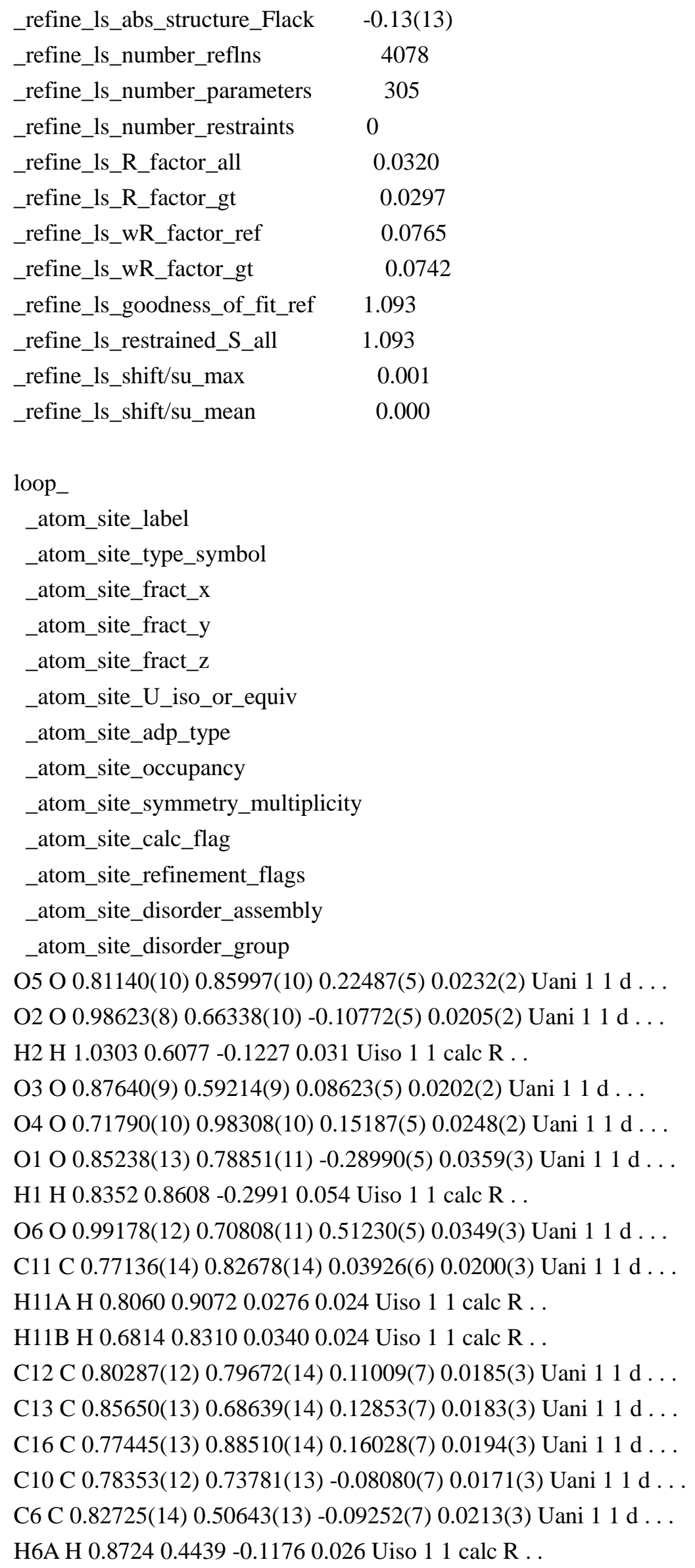


H6B H $0.73860 .4894-0.09690 .026$ Uiso 11 calc R . .

C7 C 0.86445(15) 0.49759(14) -0.01909(7) 0.0221(3) Uani $11 \mathrm{~d} \ldots$ H7A H $0.84090 .4164-0.00220 .027$ Uiso 11 calc R . .

H7B H $0.95450 .5049-0.01550 .027$ Uiso 11 calc R . .

C5 C 0.85581(12) 0.63549(13) -0.12122(7) 0.0174(3) Uani $11 \mathrm{~d}$. . C18 C 1.00540(14) 0.67575(15) 0.33420(7) 0.0267(3) Uani $11 \mathrm{~d} .$. H18 H 1.05600 .63580 .30330 .032 Uiso 11 calc R .

C3 C 0.87388(14) 0.77783(14) -0.21995(7) 0.0228(3) Uani $11 \mathrm{~d} \ldots$ H3 H $0.96350 .7888-0.21190 .027$ Uiso 11 calc R . .

C1 C 0.82161(14) 0.86702(13) -0.10703(7) 0.0199(3) Uani $11 \mathrm{~d} \ldots$ H1A H $0.77160 .9300-0.08510 .024$ Uiso 11 calc R . .

H1B H $0.90850 .8823-0.09600 .024$ Uiso 11 calc R . .

C14 C 0.89559(13) 0.66545(14) 0.19521(7) 0.0207(3) Uani $11 \mathrm{~d}$. . H14 H 0.93680 .59230 .20660 .025 Uiso 11 calc R . .

C15 C 0.87229(13) 0.75250(14) 0.24147(7) 0.0210(3) Uani $11 \mathrm{~d} \ldots$ C9 C 0.82479(12) 0.72651(13) -0.00687(7) 0.0160(3) Uani $11 \mathrm{~d}$. . H9 H 0.91550 .7393 -0.0068 0.019 Uiso 11 calc R . .

C20 C 0.95653(16) 0.72428(15) 0.44776(7) 0.0281(4) Uani $11 \mathrm{~d}$. . . C22 C 0.82888(16) 0.80402(15) 0.35992(7) 0.0294(4) Uani $11 \mathrm{~d} \ldots$ H22 H 0.76100 .85160 .34620 .035 Uiso 11 calc R . .

C8 C 0.80402(13) 0.59673(13) 0.02332(7) 0.0189(3) Uani $11 \mathrm{~d}$. . C2 C 0.80458(15) 0.87886(14) -0.18231(7) 0.0238(3) Uani $11 \mathrm{~d} \ldots$ H2A H $0.71630 .8741-0.19290 .029$ Uiso 11 calc R . .

H2B H $0.83480 .9596-0.19660 .029$ Uiso 11 calc R . .

C25 C 0.64021(12) 0.72644(15) -0.08679(7) 0.0236(3) Uani $11 \mathrm{~d} \ldots$ H25A H $0.60160 .7618-0.04810 .035$ Uiso 11 calc R . .

H25B H $0.61750 .6403-0.09040 .035$ Uiso 11 calc $R$. .

H25C H 0.61240 .7702 -0.1256 0.035 Uiso 11 calc R . .

C17 C 0.90333(14) 0.74490(14) 0.31249(7) 0.0232(3) Uani $11 \mathrm{~d} .$. C19 C 1.03261(15) 0.66564(16) 0.40107(7) 0.0290(3) Uani $11 \mathrm{~d} \ldots$ H19 H 1.10160 .61980 .41490 .035 Uiso 11 calc R . .

C23 C 0.70584(15) 0.61166(16) -0.22280(7) 0.0274(4) Uani $11 \mathrm{~d}$. . H23A H $0.70670 .6018-0.27010 .041$ Uiso 11 calc $R$. .

H23B H $0.64910 .6772-0.21100 .041$ Uiso 11 calc $R$. .

H23C H $0.67930 .5354-0.20240 .041$ Uiso 11 calc R . .

C24 C 0.92700(16) 0.55435(15) -0.23457(8) 0.0292(4) Uani 11 d . . H24A H $0.90550 .4703-0.22330 .044$ Uiso 11 calc R . .

H24B H $1.01150 .5708-0.22120 .044$ Uiso 11 calc R . .

H24C H 0.91950 .5658 -0.2817 0.044 Uiso 11 calc R . .

C21 C 0.85463(17) 0.79278(16) 0.42704(7) 0.0321(4) Uani $11 \mathrm{~d} \ldots$ H21 H 0.80330 .83130 .45810 .039 Uiso 11 calc R . .

C4 C 0.83788(13) 0.64466(14) -0.19848(7) 0.0206(3) Uani $11 \mathrm{~d}$. . . C32 C 0.66970(14) 0.56441(15) 0.04276(8) 0.0260(3) Uani $11 \mathrm{~d} \ldots$ H32A H 0.67030 .49790 .07460 .039 Uiso 11 calc R . . 
H32B H 0.62420 .53890 .00400 .039 Uiso 11 calc R .

H32C H 0.63030 .63620 .06190 .039 Uiso 11 calc R . .

C26 C 0.9144(2) 0.76188(18) 0.56253(8) 0.0413(5) Uani $11 \mathrm{~d}$...

H26A H 0.91210 .85050 .55690 .062 Uiso 11 calc R . .

H26B H 0.94800 .74240 .60550 .062 Uiso 11 calc R. .

H26C H 0.83140 .72890 .55890 .062 Uiso 11 calc R .

loop_
_atom_site_aniso_label
_atom_site_aniso_U_11
_atom_site_aniso_U_22
_atom_site_aniso_U_33
_atom_site_aniso_U_23
_atom_site_aniso_U_13
_atom_site_aniso_U_12

O5 0.0290(5) 0.0249(5) 0.0156(5) -0.0019(4) 0.0012(4) 0.0035(5)

O2 0.0138(5) 0.0261(5) 0.0216(5) -0.0042(4) -0.0002(4) 0.0029(4)

O3 0.0256(5) 0.0202(5) 0.0147(5) -0.0005(4) -0.0007(4) 0.0025(4)

O4 0.0294(6) 0.0240(5) 0.0210(5) -0.0029 (4) 0.0001(4) 0.0045(5)

O1 0.0644(8) 0.0290(6) 0.0144(5) 0.0023(5) 0.0016(5) 0.0094(6)

O6 0.0514(7) 0.0399(7) 0.0134(5) 0.0006(5) -0.0016(5) -0.0095(6)

C11 0.0225(7) 0.0216(7) 0.0158(7) -0.0012(6) 0.0001(5) 0.0035(6)

C12 0.0169(7) 0.0222(7) 0.0163(7) -0.0004(6) 0.0026(5) -0.0015(6)

C13 0.0157(6) 0.0223(7) 0.0168(7) -0.0009(6) 0.0035(5) -0.0025(6)

C16 0.0181(6) 0.0240(7) 0.0162(7) 0.0006(6) 0.0008(5) -0.0009(6)

C10 0.0151(6) 0.0203(7) 0.0159(7) -0.0014(6) -0.0005(5) 0.0021(6)

C6 0.0260(7) 0.0198(7) 0.0181(7) -0.0029(6) -0.0010(6) 0.0004(6)

C7 0.0299(8) 0.0184(7) 0.0180(7) -0.0001(6) -0.0003(6) 0.0009(6)

C5 0.0147(7) 0.0207(7) 0.0168(7) -0.0021(6) -0.0016(5) -0.0006(6)

C18 0.0282(8) 0.0341(9) 0.0177(7) -0.0011(7) 0.0028(6) -0.0012(7)

C3 0.0295(8) 0.0259(8) 0.0130(7) 0.0018(6) 0.0003(6) 0.0012(7)

C1 0.0231(7) 0.0193(7) 0.0173(7) -0.0011(6) -0.0009(6) 0.0025(6)

C14 0.0211(7) 0.0239(8) 0.0171(7) 0.0025(6) 0.0021(5) -0.0009(6)

C15 0.0200(7) 0.0238(7) 0.0193(7) 0.0030(6) 0.0024(6) -0.0009(6)

C9 0.0141(6) 0.0184(7) 0.0155(7) -0.0020(6) -0.0005(5) 0.0000(6)

C20 0.0407(9) 0.0275(8) 0.0160(7) 0.0003(6) -0.0003(6) -0.0113(8)

C22 0.0381(9) 0.0271(8) 0.0229(8) 0.0009(7) 0.0030(7) 0.0051(7)

C8 0.0214(7) 0.0217(7) 0.0138(7) 0.0008(6) -0.0022(6) -0.0009(6)

C2 0.0324(8) 0.0200(7) 0.0189(8) 0.0020(6) 0.0004(6) 0.0028(6)

C25 0.0156(7) 0.0328(8) 0.0223(7) -0.0019(7) -0.0009(6) 0.0027(6)

C17 0.0280(8) 0.0254(8) 0.0162(7) 0.0000(6) 0.0017(6) -0.0036(6)

C19 0.0305(8) 0.0356(9) 0.0208(8) 0.0027(7) -0.0021(6) -0.0014(7)

C23 0.0316(8) 0.0305(8) 0.0203(8) -0.0031(7) -0.0079(6) -0.0025(7)

C24 0.0393(9) 0.0304(9) 0.0179(8) -0.0035(7) 0.0014(7) 0.0111(8) 
C21 0.0500(11) 0.0302(9) 0.0162(8) -0.0048(7) 0.0087(7) 0.0029(8)

C4 0.0229(7) 0.0229(8) 0.0159(7) -0.0030(6) -0.0008(5) 0.0028(6)

C32 0.0266(8) 0.0272(8) 0.0241(8) 0.0003(7) 0.0027(6) -0.0072(7)

C26 0.0660(13) 0.0410(10) 0.0171(8) -0.0058(8) 0.0086(8) -0.0185(9)

_geom_special_details

;

All esds (except the esd in the dihedral angle between two l.s. planes) are estimated using the full covariance matrix. The cell esds are taken into account individually in the estimation of esds in distances, angles and torsion angles; correlations between esds in cell parameters are only used when they are defined by crystal symmetry. An approximate (isotropic) treatment of cell esds is used for estimating esds involving l.s. planes.

;

loop_

_geom_bond_atom_site_label_1

_geom_bond_atom_site_label_2

_geom_bond_distance

_geom_bond_site_symmetry_2

_geom_bond_publ_flag

O5 C15 1.3681(18) . ?

O5 C16 1.3867(16) . ?

$\mathrm{O} 2 \mathrm{C} 5$ 1.4532(16) . ?

$\mathrm{O} 2 \mathrm{H} 20.8200$. ?

O3 C13 1.3412(18) . ?

O3 C8 1.4862(16) . ?

O4 C16 1.2267(18) . ?

O1 C3 1.4326(17) . ?

O1 H1 0.8200 . ?

O6 C20 1.3651(18) . ?

O6 C26 1.430(2) . ?

C11 C12 1.5015(19) . ?

C11 C9 1.5339(19) . ?

C11 H11A 0.9700 . ?

C11 H11B 0.9700 . ?

C12 C13 1.369(2) . ?

C12 C16 1.421(2) . ?

C13 C14 1.425(2) . ?

C10 C1 1.5412(19) . ?

C10 C25 1.5433(18) . ?

C10 C9 1.5584(18) . ?

C10 C5 1.5722(19) . ?

C6 C5 1.534(2) . ? 
C6 C7 1.5350(19) . ?

C6 H6A 0.9700 . ?

C6 H6B 0.9700 . ?

C7 C8 1.512(2) . ?

C7 H7A 0.9700 . ?

C7 H7B 0.9700 . ?

C5 C4 1.5715(19) . ?

C18 C19 1.383(2). ?

C18 C17 1.392(2) . ?

C18 H18 0.9300 . ?

C3 C2 1.518(2) . ?

C3 C4 1.545(2) . ?

C3 H3 0.9800.?

C1 C2 1.5330(19) . ?

C1 H1A 0.9700 .?

C1 H1B 0.9700 .?

C14 C15 1.344(2) . ?

C14 H14 0.9300 .?

C15 C17 1.471(2) . ?

C9 C8 1.5384(19) . ?

C9 H9 0.9800 . ?

C20 C21 1.381(2) . ?

C20 C19 1.395(2) . ?

C22 C21 1.385(2). ?

C22 C17 1.397(2) . ?

$\mathrm{C} 22 \mathrm{H} 22$ 0.9300.?

C8 C32 1.530(2) . ?

C2 H2A 0.9700 .?

C2 H2B 0.9700 . ?

C25 H25A 0.9600 .?

C25 H25B 0.9600 .?

C25 H25C 0.9600 . ?

C19 H19 0.9300 . ?

C23 C4 1.537(2). ?

C23 H23A 0.9600 . ?

C23 H23B 0.9600 . ?

$\mathrm{C} 23 \mathrm{H} 23 \mathrm{C} 0.9600$. ?

C24 C4 1.543(2) . ?

C24 H24A 0.9600 . ?

C24 H24B 0.9600 . ?

C24 H24C 0.9600 . ?

C21 H21 0.9300 .?

C32 H32A 0.9600 . ?

C32 H32B 0.9600 . ? 
$\mathrm{C} 32 \mathrm{H} 32 \mathrm{C} 0.9600$. ?

C26 H26A 0.9600 . ?

C26 H26B 0.9600 . ?

C26 H26C 0.9600 . ?

loop_

_geom_angle_atom_site_label_1

_geom_angle_atom_site_label_2

_geom_angle_atom_site_label_3

_geom_angle

_geom_angle_site_symmetry_1

_geom_angle_site_symmetry_3

_geom_angle_publ_flag

C15 O5 C16 122.00(11) . . ?

C5 O2 H2 109.5 .. ?

C13 O3 C8 115.73(11)..?

C3 O1 H1 109.5 . . ?

C20 O6 C26 117.57(14) . . ?

C12 C11 C9 109.93(12) . . ?

C12 C11 H11A 109.7 . . ?

C9 C11 H11A 109.7 .. ?

C12 C11 H11B 109.7 .. ?

C9 C11 H11B 109.7 .. ?

H11A C11 H11B 108.2 . ?

C13 C12 C16 118.42(13) . . ?

C13 C12 C11 122.59(13) . . ?

C16 C12 C11 118.98(13) . . ?

O3 C13 C12 123.27(12) . . ?

O3 C13 C14 115.67(13) . . ?

C12 C13 C14 121.06(13) .. ?

O4 C16 O5 115.96(13) . . ?

O4 C16 C12 125.57(13) . . ?

O5 C16 C12 118.47(13) . . ?

C1 C10 C25 107.90(12) . . ?

C1 C10 C9 108.85(11) .. ?

C25 C10 C9 110.50(11)..?

C1 C10 C5 108.84(11) .. ?

C25 C10 C5 113.14(12). . ?

C9 C10 C5 107.53(11) .. ?

C5 C6 C7 111.57(12) . . ?

C5 C6 H6A 109.3 .. ?

C7 C6 H6A 109.3 . . ?

C5 C6 H6B 109.3 .. ?

C7 C6 H6B 109.3 . . ? 
H6A C6 H6B 108.0 . . ?

C8 C7 C6 112.97(12) . . ?

C8 C7 H7A 109.0 . . ?

C6 C7 H7A 109.0 . . ?

C8 C7 H7B 109.0 . . ?

C6 C7 H7B 109.0 . . ?

H7A C7 H7B 107.8 . . ?

O2 C5 C6 107.90(11) . . ?

O2 C5 C4 106.84(11) . . ?

C6 C5 C4 113.94(12) . . ?

O2 C5 C10 103.37(10) . . ?

C6 C5 C10 109.85(11) . . ?

C4 C5 C10 114.16(11) .. ?

C19 C18 C17 120.90(14) . . ?

C19 C18 H18 119.6 . . ?

C17 C18 H18 119.6 . . ?

O1 C3 C2 110.83(12) . . ?

O1 C3 C4 108.05(12) . . ?

C2 C3 C4 113.67(12) . . ?

O1 C3 H3 108.0 . . ?

C2 C3 H3 108.0 . . ?

C4 C3 H3 108.0 . . ?

C2 C1 C10 112.50(12) . . ?

C2 C1 H1A 109.1 .. ?

C10 C1 H1A 109.1 . . ?

C2 C1 H1B 109.1 ..?

C10 C1 H1B 109.1 . . ?

H1A C1 H1B 107.8 . . ?

C15 C14 C13 119.29(14) . . ?

C15 C14 H14 120.4 . . ?

C13 C14 H14 120.4 . . ?

C14 C15 O5 120.46(13) . . ?

C14 C15 C17 126.43(14) . . ?

O5 C15 C17 113.10(12) . . ?

C11 C9 C8 110.13(11) .. ?

C11 C9 C10 114.76(11) . . ?

C8 C9 C10 114.06(11) . . ?

C11 C9 H9 105.7 . . ?

C8 C9 H9 105.7 . . ?

C10 C9 H9 105.7 . . ?

O6 C20 C21 125.06(15) . . ?

O6 C20 C19 115.06(15) . . ?

C21 C20 C19 119.88(14). . ?

C21 C22 C17 120.97(15) . . ? 


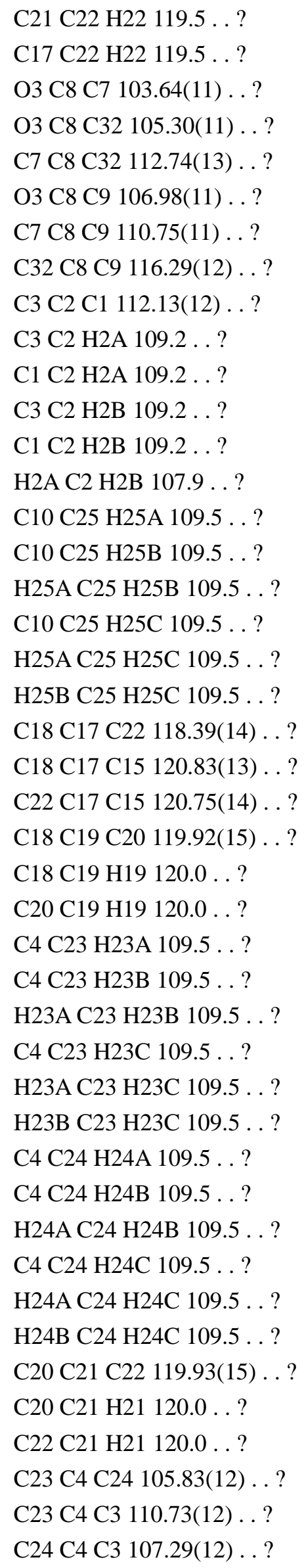


C23 C4 C5 114.42(12) ..?

C24 C4 C5 110.60(12) .. ?

C3 C4 C5 107.76(11) . . ?

C8 C32 H32A 109.5 . . ?

C8 C32 H32B 109.5 . . ?

H32A C32 H32B 109.5 .. ?

C8 C32 H32C 109.5 . . ?

H32A C32 H32C 109.5 . . ?

H32B C32 H32C 109.5 . . ?

O6 C26 H26A 109.5 . . ?

O6 C26 H26B 109.5 . . ?

H26A C26 H26B 109.5 . . ?

O6 C26 H26C 109.5 . . ?

H26A C26 H26C 109.5 . . ?

H26B C26 H26C 109.5 . . ?

loop_

_geom_torsion_atom_site_label_1

_geom_torsion_atom_site_label_2

_geom_torsion_atom_site_label_3

_geom_torsion_atom_site_label_4

_geom_torsion

_geom_torsion_site_symmetry_1

_geom_torsion_site_symmetry_2

_geom_torsion_site_symmetry_3

_geom_torsion_site_symmetry_4

_geom_torsion_publ_flag

C9 C11 C12 C13 -8.07(19) ... . ?

C9 C11 C12 C16 172.89(12) ....?

C8 03 C13 C12 -17.84(18) ... . ?

C8 O3 C13 C14 162.46(11) ... . ?

C16 C12 C13 O3 173.57(13) ... ? ?

$\mathrm{C} 11 \mathrm{C} 12 \mathrm{C} 13 \mathrm{O} 3-5.5(2) \ldots$ ?

C16 C12 C13 C14 -6.7(2) .... ?

C11 C12 C13 C14 174.20(13) ....?

C15 O5 C16 O4 178.27(12) ... . ?

C15 O5 C16 C12 -0.96(19) .... ?

C13 C12 C16 O4 -174.02(14) ... . ?

C11 C12 C16 O4 5.1(2) ....?

C13 C12 C16 O5 5.1(2) ....?

$\mathrm{C} 11 \mathrm{C} 12 \mathrm{C} 16 \mathrm{O} 5$-175.79(12)....?

C5 C6 C7 C8 55.66(17) ... . ?

C7 C6 C5 O2 53.23(15) ....?

C7 C6 C5 C4 171.69(11) .... ? 


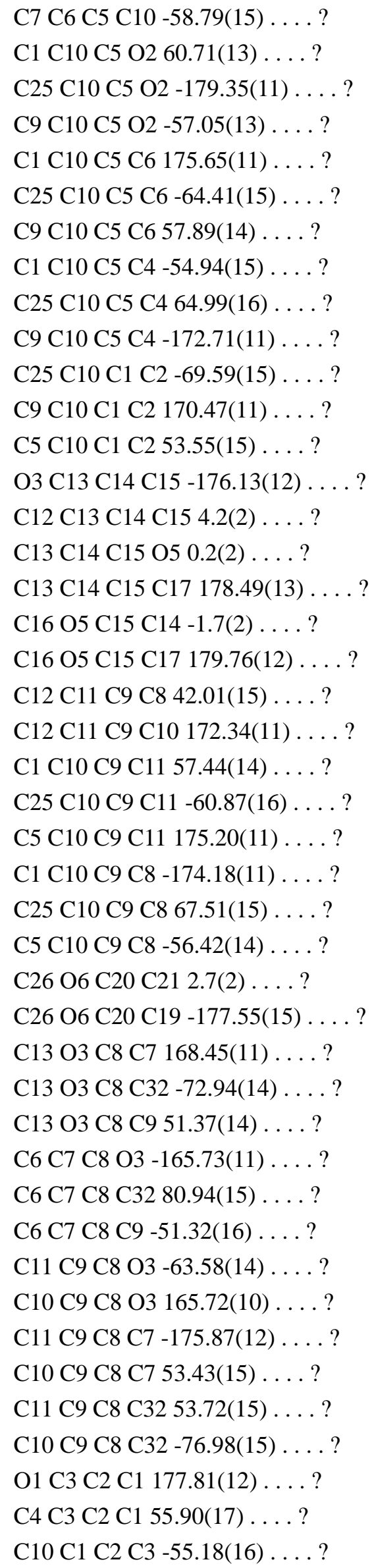




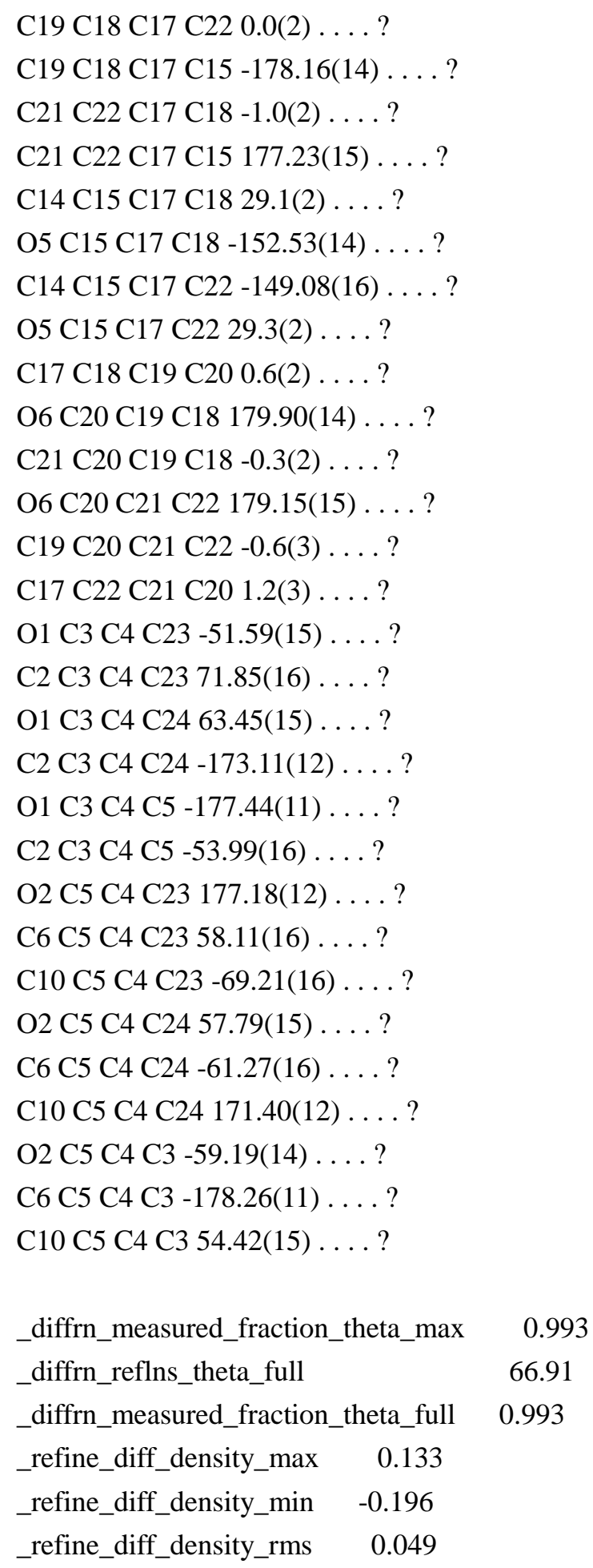

